\title{
Theoretical framework for the atomistic modeling of frequency-dependent liquid-solid friction
}

\author{
Haruki Oga $\odot,{ }^{1, *}$ Takeshi Omori $\odot,{ }^{2, \dagger}$ Cecilia Herrero $\odot,{ }^{3}$ Samy Merabia $\odot,{ }^{3}$ Laurent Joly $\odot,{ }^{3,4}$ and Yasutaka Yamaguchi $\odot^{1,5, \hbar}$ \\ ${ }^{1}$ Department of Mechanical Engineering, Osaka University, 2-1 Yamadaoka, Suita, Osaka 565-0871, Japan \\ ${ }^{2}$ Department of Mechanical Engineering, Osaka City University, 3-3-138 Sugimoto, Sumiyoshi, Osaka, Osaka 558-8585, Japan \\ ${ }^{3}$ Univ Lyon and Université Claude Bernard Lyon 1, CNRS, Institut Lumière Matière, F-69622 Villeurbanne, France \\ ${ }^{4}$ Institut Universitaire de France (IUF), 1 rue Descartes, 75005 Paris, France \\ ${ }^{5}$ Water Frontier Research Center (WaTUS), Research Institute for Science \& Technology, \\ Tokyo University of Science, 1-3 Kagurazaka, Shinjuku-ku, Tokyo 162-8601, Japan
}

(Received 9 April 2021; accepted 7 July 2021; published 27 July 2021)

\begin{abstract}
Nanofluidics shows great promise for energy conversion and desalination applications. The performance of nanofluidic devices is controlled by liquid-solid friction, quantified by the Navier friction coefficient (FC). Despite decades of research, there is no well-established generic framework to determine the frequency-dependent Navier FC from atomistic simulations. Here, we have derived analytical expressions to connect the Navier FC to the random force autocorrelation on the confining wall, from the observation that the random force autocorrelation can be related to the hydrodynamic boundary condition, where the Navier FC appears. The analytical framework is generic in the sense that it explicitly includes the system size dependence and also the frequency dependence of the FC, which enabled us to address (i) the long-standing plateau issue in the evaluation of the FC and (ii) the non-Markovian behavior of liquid-solid friction of a Lennard-Jones liquid and of water on various walls and at various temperatures, including the supercooled regime. This framework opens the way to explore the frequency-dependent FC for a wide range of complex liquids.
\end{abstract}

DOI: 10.1103/PhysRevResearch.3.L032019

Introduction. Nanofluidics is the discipline that describes fluid motion in nanoconfinement, whose unique behavior in the mass and ionic transport should be a key ingredient in future technologies for fluid filtration and energy harvesting [1-5]. The recent advent of new materials and fabrication techniques to create fine fluid conduits [6] has even increased the importance of exploring nanofluidic transport. In nanofluidic systems, surface effects play a critical role because of the large surface-to-volume ratio. In particular, liquid-solid slip can boost the performance of nanofluidic devices [7-10].

Liquid-solid slip was first foreseen by Navier [11], who proposed that the slip velocity $u_{\text {slip }}$ is proportional to the shear stress on the wall $\tau$ as

$$
\tau=\lambda u_{\text {slip }}
$$

with $\lambda$ being the Navier friction coefficient (FC). The Navier boundary condition, Eq. (1), has been tested by many authors in the past decades as reviewed in Refs. [2,12-14]. From the theoretical side, the pioneering work by Bocquet and Barrat $[15,16]$ showed that $\lambda$ can be related to the equilibrium

\footnotetext{
*haruki@nnfm.mech.eng.osaka-u.ac.jp

†omori@osaka-cu.ac.jp

†yamaguchi@mech.eng.osaka-u.ac.jp
}

Published by the American Physical Society under the terms of the Creative Commons Attribution 4.0 International license. Further distribution of this work must maintain attribution to the author(s) and the published article's title, journal citation, and DOI. fluctuations of the friction force through a Green-Kubo (GK) formula:

$$
\lambda=\lim _{t \rightarrow \infty} \frac{1}{S k_{\mathrm{B}} T} \int_{0}^{t}\left\langle\delta F\left(t^{\prime}\right) \delta F(0)\right\rangle d t^{\prime},
$$

where $\delta F$ is the random friction force on the wall at equilibrium, $S$ is the wall surface area, and $T$ is the temperature, with $k_{\mathrm{B}}$ being the Boltzmann constant. Later, the system size dependence of the formula, called the plateau problem, was pointed out [17], and alternative ways to estimate the FC have been proposed [18-21].

Recently, two theoretical approaches have been put forward to challenge the plateau problem fundamentally. Español and co-workers [22,23] developed a new theory of nonequilibrium statistical mechanics, which led to a corrected form of the GK formula under the assumption that the system is Markovian. In other recent work, Nakano and Sasa [24,25] introduced explicit assumptions on the scale separation between the microscopic motion of molecules and the macroscopic motion of fluid and proposed a new way to estimate $\lambda$ based on linearized fluctuating hydrodynamics. These works from the two groups involved elaborate mathematical manipulations and only reported the pure viscous (Markovian) behavior of the Navier FC, for a Lennard-Jones (LJ) liquid on a simple model wall. However, non-Markovian behavior of the FC was recently reported for a $\mathrm{LJ}$ liquid on a fcc lattice [26], and it is plausible that more complex liquids such as water also show such behavior, in analogy with their bulk transport properties [27-32]. 
(a)

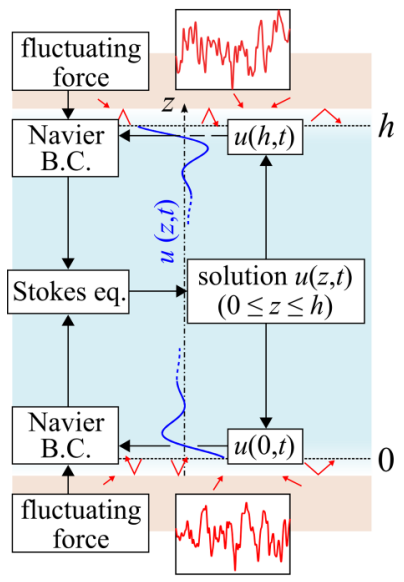

(c)

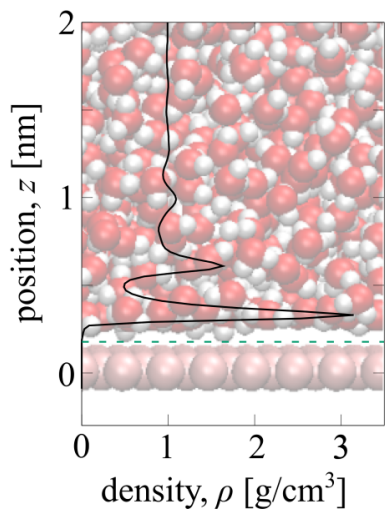

(b)

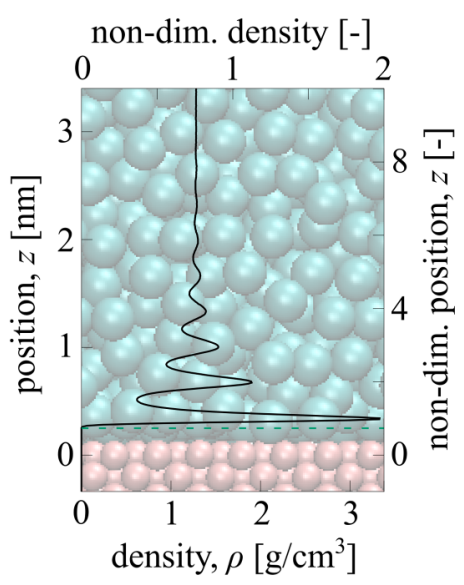

(d)

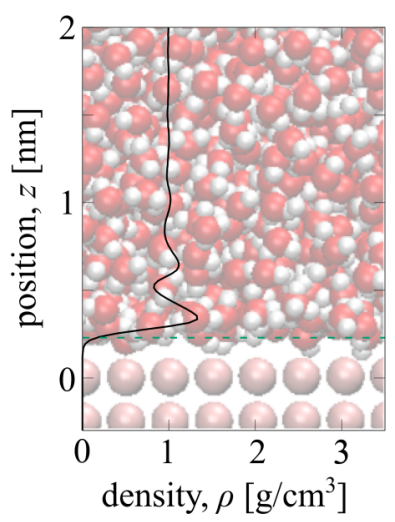

FIG. 1. (a) Model system for the derivation of the Navier friction coefficient, and (b)-(d) snapshots of the bottom half of the systems tested in this study overlaid with density distributions: (b) LennardJones liquid confined between fcc walls, (c) water confined between graphene walls, and (d) water confined between fcc walls. The dashed green lines denote the position of the hydrodynamic boundaries. For (b), the system with a contact angle of $79^{\circ}$ is shown. We give the details on the system dimensions in the Supplemental Material [33]. B.C., boundary condition; non-dim., nondimensional.

In this Research Letter, we develop a theory to relate the Navier FC and the random force autocorrelation on the wall, by employing rather classical tools such as Stokes and Langevin equations. This theoretical framework offers some perspectives on long-standing debates related to the GK modeling of liquid-solid friction, together with a simple and fast method to fully characterize the frequency-dependent Navier FC. We then apply this method to explore the frictional behavior of a simple LJ liquid and of water at various temperatures-including the supercooled regime.

Theory. Let us consider the system shown in Fig. 1(a), where a liquid is confined between two solid walls under no external field. When the bottom wall is allowed to move freely in a wall-tangential direction $x$, its motion can be described by a Langevin equation [34]:

$$
M \frac{d U}{d t}=-S \int_{0}^{t} \xi\left(t-t^{\prime}\right) U\left(t^{\prime}\right) d t^{\prime}+\delta F^{\mathrm{bot}},
$$

where $M, S$, and $U$ are the mass, the surface area, and the $x$-direction velocity of the bottom wall, respectively; $\xi$ is the friction kernel, and $\delta F^{\text {bot }}$ is the random force that originates from the direct interaction between the solid and liquid particles. Assuming the equipartition of energy, Eq. (3) leads to the fluctuation-dissipation theorem:

$$
C_{\delta F^{\text {bot }}}(t):=\left\langle\delta F^{\text {bot }}(t) \delta F^{\text {bot }}(0)\right\rangle=S k_{\mathrm{B}} T \xi(t) .
$$

The motion of the liquid in response to the bottom wall motion (the top wall is fixed) can be described by the Stokes equation for a wide frequency range [26,35]:

$$
\frac{\partial u(z, t)}{\partial t}=\frac{\eta}{\rho} \frac{\partial^{2} u(z, t)}{\partial z^{2}},
$$

with the Navier boundary condition defined on the bottom and top hydrodynamic boundaries

$$
\begin{aligned}
& \left.\eta \frac{\partial u(z, t)}{\partial z}\right|_{z=0}=\int_{0}^{t} \lambda\left(t-t^{\prime}\right)\left[u\left(0, t^{\prime}\right)-U\left(t^{\prime}\right)\right] d t^{\prime} \\
& \left.\eta \frac{\partial u(z, t)}{\partial z}\right|_{z=h}=\int_{0}^{t} \lambda\left(t-t^{\prime}\right)\left[-u\left(h, t^{\prime}\right)\right] d t^{\prime},
\end{aligned}
$$

where $u, t, \rho, \eta$, and $\lambda$ denote the liquid velocity in the $x$ direction, the time, the bulk liquid density, the bulk liquid viscosity, and the Navier FC, respectively. Note that $\lambda$ is frequency dependent and of non-Markovian nature: $\lambda$ has the dimension $[\mathrm{Pa} / \mathrm{m}]$ instead of $[\mathrm{Pa} \cdot \mathrm{s} / \mathrm{m}]$ adequate for the frequency-independent FC. Because the first term on the righthand side of Eq. (3) would also be written as $-S \int_{0}^{t} \lambda(t-$ $\left.t^{\prime}\right)\left[U\left(t^{\prime}\right)-u\left(0, t^{\prime}\right)\right] d t^{\prime}$ in terms of the slip velocity on the wall, the friction kernel $\xi$ is given from the solution of Eqs. (5) and (6) (for the complete derivation, see the Supplemental Material [33]). Combined with Eq. (4), the expression for the force autocorrelation function is obtained:

$$
\frac{\tilde{C}_{\delta F} \text { bot }}{S k_{\mathrm{B}} T}=\frac{\tilde{\lambda} \eta \zeta[\eta \zeta \sinh (\zeta h)+\tilde{\lambda} \cosh (\zeta h)]}{\left(\tilde{\lambda}^{2}+\eta^{2} \zeta^{2}\right) \sinh (\zeta h)+2 \tilde{\lambda} \eta \zeta \cosh (\zeta h)},
$$

where the tilde indicates that the variables are Fourier-Laplace transformed and $\zeta$ denotes $\sqrt{i \rho \omega / \eta}$, with $\omega$ being the angular frequency. Considering that the nature of the random force is independent of the wall velocity by construction, Eq. (7) holds even when the bottom wall is fixed. Unlike the GK formula by Bocquet and Barrat [34], this equation explicitly includes the system size dependence in the relation between the random force autocorrelation and the Navier FC. It is also more general as it provides the viscoelastic behavior of the friction coefficient. From Eq. (7), the asymptotic behaviors of the random force autocorrelation are

$$
\begin{aligned}
\lim _{\omega \rightarrow 0, h: \text { finite }} \frac{\tilde{C}_{\delta F} \text { bot }}{S k_{\mathrm{B}} T} & =\frac{\lambda_{0}}{h / b+2}, \\
\lim _{h \rightarrow \infty, \omega: \text { finite }} \frac{\tilde{C}_{\delta F^{\text {bot }}}}{S k_{\mathrm{B}} T} & =\frac{\tilde{\lambda}}{\tilde{\lambda} /(\eta \zeta)+1},
\end{aligned}
$$

where $\lambda_{0}$ is the zero-frequency component of the Navier FC and $b$ is the slip length defined as $\eta / \lambda_{0}$.

From Eqs. (8) and (9), one can derive several important properties of the random force, the evidence for which will be shown later in the Results section. First, the GK integral of the random force [Eq. (8)] is not zero but has a finite value 
that depends on the system height $h$, which tells us that the integral has a plateau. Note that the Bocquet-Barrat formula may be recovered by taking the plug-flow limit $b \gg h$. In this limit only, the GK integral no longer depends on the system height $h$. Second, in the thermodynamic limit where the system height is infinite, the GK integral of the random force goes to zero: This is true regardless of the order in which the limits are taken, $\lim _{h \rightarrow \infty} \lim _{\omega \rightarrow 0} \tilde{C}_{\delta F}$ bot $=\lim _{\omega \rightarrow 0} \lim _{h \rightarrow \infty} \tilde{C}_{\delta F}$ bot $=$ 0 . Finally, when the frequency $\omega$ is high enough so that the penetration length is much smaller than the magnitude of the complex slip length $(\sqrt{\eta / \rho \omega} \ll|\eta / \tilde{\lambda}|)$ as well as much smaller than the system height $(\sqrt{\eta / \rho \omega} \ll h)$, the random force autocorrelation coincides with the Navier FC:

$$
\frac{C_{\delta F \text { bot }}(t)}{S k_{\mathrm{B}} T} \approx \lambda(t)
$$

for small $t$ satisfying $\sqrt{\eta t / \rho} \ll \min \{|\eta / \tilde{\lambda}|, h\}$. All these results, which are in contrast to the common view that $\lambda_{0}$ might be obtained as $\lim _{\omega \rightarrow 0} \lim _{h \rightarrow \infty} \tilde{C}_{\delta F}$ bot $[34,36]$, reflect our explicit consideration of the system height and its hydrodynamic influence on the fluctuations of the friction force.

Interestingly, one can find the Navier FC for the whole frequency range from the measured random force autocorrelation $C_{\delta F}$ bot by solving Eq. (7) for $\tilde{\lambda}$, which is a quadratic equation of it. The physically correct solution out of the two can be chosen so that it satisfies the following relationship:

$$
\frac{\tilde{C}_{\delta F^{\text {total }}}}{2 S k_{\mathrm{B}} T}=\frac{\tilde{\lambda} \eta \zeta\{\eta \zeta \sinh (\zeta h)+\tilde{\lambda}[\cosh (\zeta h)-1]\}}{\left(\tilde{\lambda}^{2}+\eta^{2} \zeta^{2}\right) \sinh (\zeta h)+2 \tilde{\lambda} \eta \zeta \cosh (\zeta h)},
$$

where $C_{\delta F^{\text {total }}}$ is the autocorrelation function of the random force summed over both the top and bottom walls (for the complete procedure, see the Supplemental Material [33]).

Simulation. To validate the ideas in the Theory section, we performed equilibrium molecular dynamics (MD) simulations for two kinds of liquid on different walls.

The first system was a LJ liquid confined between two fcc crystal walls [Fig. 1(b)]. The liquid consisted of 6400 molecules unless otherwise mentioned. The quantities for this system are shown in LJ reduced units based on the liquid molecular mass $m_{\mathrm{f}}\left(=6.634 \times 10^{-26} \mathrm{~kg}\right)$ and two parameters $\sigma_{\mathrm{ff}}(=3.40 \AA)$ and $\varepsilon_{\mathrm{ff}}\left(=1.67 \times 10^{-21} \mathrm{~J}\right)$ for the $\mathrm{LJ}$ potential $\Phi_{\mathrm{LJ}}\left(r_{i j}\right)=4 \varepsilon\left[\left(\sigma / r_{i j}\right)^{12}-\left(\sigma / r_{i j}\right)^{6}\right]$ between the liquid molecules, with $r_{i j}$ being the distance between particles $i$ and $j$ with a cutoff distance of $3.50 \sigma$ [37]. Each solid wall consisted of eight layers of atoms in the (001) plane of a fcc crystal with a lattice constant of $1.15 \sigma_{\mathrm{ff}}$. The system temperature was controlled at $0.827 \varepsilon_{\mathrm{ff}} / k_{\mathrm{B}}$ by a Langevin thermostat set on the second outermost layer of the walls, and the pressure was set to $0.094 \varepsilon_{\mathrm{ff}} / \sigma_{\mathrm{ff}}^{3}$ by a preliminary piston equilibration (see Ref. [26] for technical details). For the liquid-solid interaction, the LJ potential was adopted as well. To see the effect of the wettability, which is known to have an impact on the friction [2], three different $\varepsilon_{\mathrm{fw}}$ were used, $\varepsilon_{\mathrm{fw}}=\varepsilon_{\mathrm{fw}}^{0}=0.155 \varepsilon_{\mathrm{ff}}, \varepsilon_{\mathrm{fw}}=2 \varepsilon_{\mathrm{fw}}^{0}$, and $\varepsilon_{\mathrm{fw}}=3 \varepsilon_{\mathrm{fw}}^{0}$, while $\sigma_{\mathrm{fw}}=1.01 \sigma_{\mathrm{ff}}$ was kept constant. The corresponding contact angles of a sessile LJ droplet on the three walls were $136^{\circ}$, $79^{\circ}$, and $0^{\circ}$, respectively [38].

The other two systems were water confined between either graphene walls or fcc crystal walls [Figs. 1(c) and 1(d), respectively]. In this case the fluid was constituted of 4096

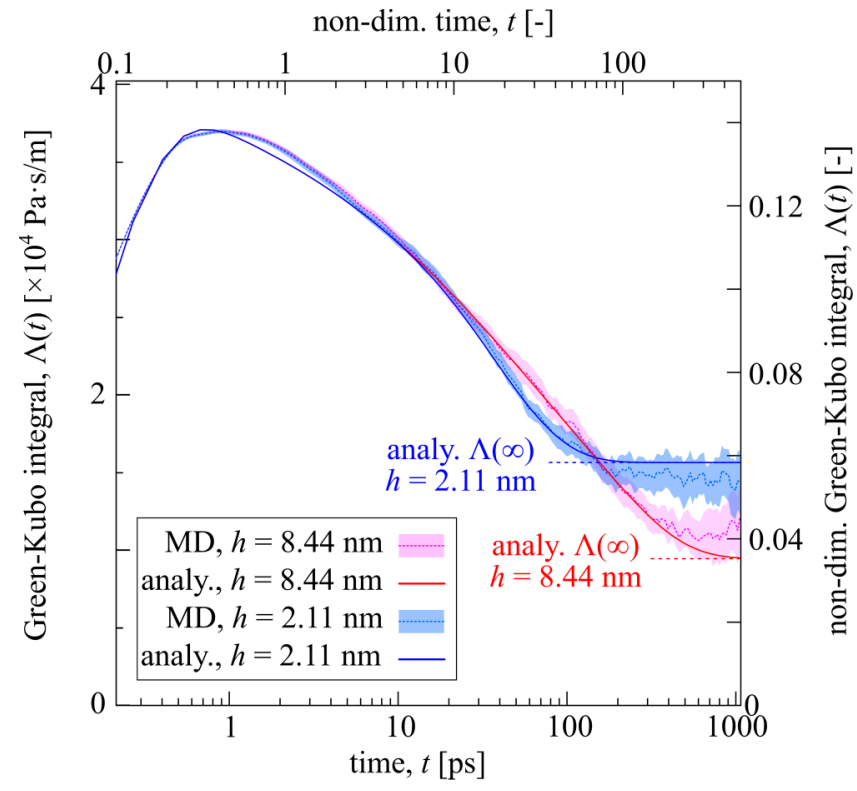

FIG. 2. Size dependence of the finite-time GK integral for the system in Fig. 1(b): comparison between analytical (analy.) predictions and MD results for two system heights $h$. The liquid consisted of 6400 and 1600 molecules for $h=8.44$ and $2.11 \mathrm{~nm}$, respectively.

TIP4P/2005 water molecules [39]. For water enclosed between generic fcc walls, such walls were constituted of three atomic layers of a fcc crystal exposing the (001) face with a lattice constant of $5.356 \AA$ and with liquid-solid interaction parameters taken for hydrophobic walls from Ref. [40]. For water enclosed between graphene walls, the liquid-solid interaction parameters were taken from Ref. [41]. For both systems, the temperature was controlled by applying a NoséHoover thermostat to liquid atoms, and the pressure was set to 1 atm through a preliminary piston equilibration (see Ref. [42] for technical details).

Results. First, we discuss the convergence of the GK integral of the random force autocorrelation. Figure 2 shows the normalized finite-time GK integral, $\Lambda(t):=$ $\int_{0}^{t} C_{\delta F}$ bot $\left(t^{\prime}\right) d t^{\prime} / S k_{\mathrm{B}} T$, as a function of the upper time limit of the integration, for the LJ liquid confined by walls with

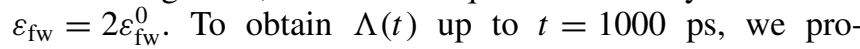
duced the simulation data typically for $300 \mathrm{~ns}$. One can see that $\Lambda(t)$ has a system-height-dependent plateau for $t \rightarrow$ $\infty$, whose value, the GK integral, decreases by increasing the system height. The figure also illustrates that the whole $\Lambda(t)$ profile is well reproduced with $C_{\delta F}$ bot calculated from the right-hand side of Eq. (7), and the plateau values coincide with the right-hand side of Eq. (8). In this evaluation of Eqs. (7) and (8), we substituted $\lambda(t)$ by the Maxwell-type model $\lambda_{0} \exp \left(-t / t_{\lambda}\right) / t_{\lambda}$ with the parameters $\lambda_{0}=0.1492 \sqrt{m_{\mathrm{f}} \varepsilon_{\mathrm{ff}}} / \sigma_{\mathrm{ff}}^{3}$ and $t_{\lambda}=0.077 \sigma_{\mathrm{ff}} \sqrt{m_{\mathrm{f}} / \varepsilon_{\mathrm{ff}}}$ taken from the results of nonequilibrium simulations [26], whose simulation system and conditions were identical to those of the present study [43]. Here, the same $\lambda$ was used regardless of the system height: This shows that there is no system size dependence in the estimation of $\lambda$ from the MD simulation data by the present theory. Sometimes in the literature $[16,44], \lambda_{0}$ is estimated as max $\Lambda(t)$, which gives about $0.14 \sqrt{m_{\mathrm{f}} \varepsilon_{\mathrm{ff}}} / \sigma_{\mathrm{ff}}^{3}$ 
(a)

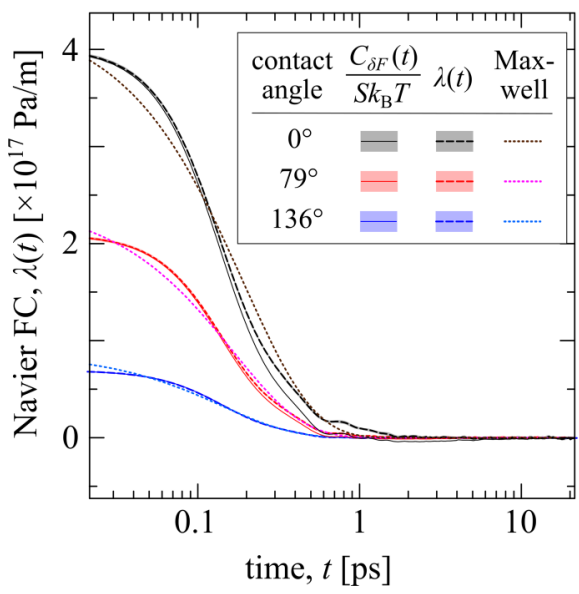

(b)

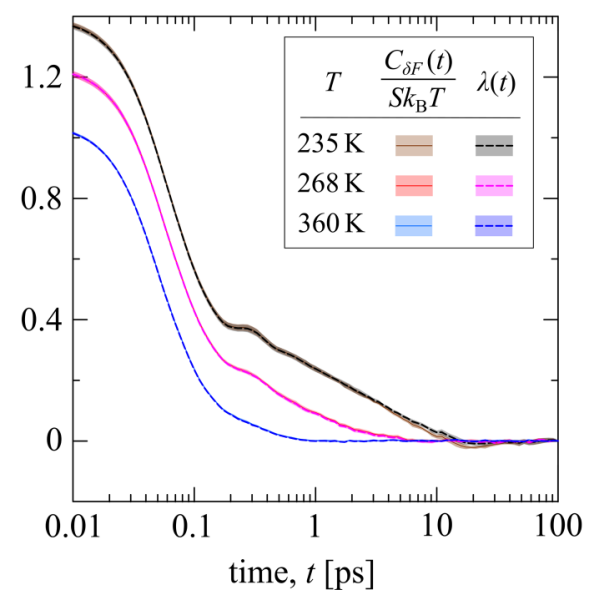

(c)

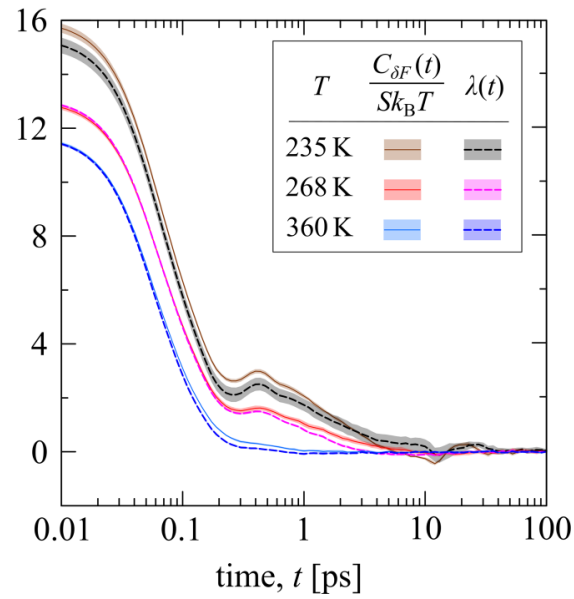

FIG. 3. Random force autocorrelation function $C_{\delta F}$ bot $(t) /\left(S k_{\mathrm{B}} T\right)$ and friction coefficient $\lambda(t)$ obtained from Eq. (7) for three different systems: (a) Lennard-Jones liquid confined between fcc walls with three different wettabilities, (b) water confined between graphene walls, and (c) water confined between fcc walls under three different temperatures. The Maxwell viscoelastic model is also shown for (a).

and slightly underestimates $\lambda_{0}$. This underestimation was also shown in Ref. [21]. In summary, the discussion here provides a perspective on the long-standing plateau issue for the evaluation of the GK integral: There is a plateau in the limit of the integral for finite-sized systems, and this plateau value has a hydrodynamic meaning.

Now we determine the complete nature of the Navier FC for the three liquid-solid interfaces shown in Fig. 1. As described in the Theory section, the Navier FC $\lambda$ can be obtained by solving Eq. (7) for $\lambda$ once the bulk liquid properties and the random force autocorrelation are measured. To estimate the hydrodynamic system height $h$, for the LJ liquid system we employed the values from Ref. [26], and for the water systems we adopted the separation between the Gibbs dividing surfaces [45] on the two confining walls.

Figure 3 shows the Navier FC $\lambda(t)$ and the normalized random force autocorrelation $C_{\delta F}$ bot $(t) / S k_{\mathrm{B}} T$ for the $\mathrm{LJ}$ liquid and for water. The equivalence between $\lambda$ and $C_{\delta F}$ bot $/ S k_{\mathrm{B}} T$, Eq. (10), holds almost everywhere, although the condition $\sqrt{\eta t / \rho} \ll \min \{|\eta / \tilde{\lambda}|, h\}$ is not strictly satisfied in the tail region for supercooled water. Figure 3(a) shows $\lambda(t)$ for the LJ liquid system, together with a Maxwell-type viscoelastic model $\lambda_{M}(t)=\lambda_{0} \exp \left(-t / t_{\lambda}\right) / t_{\lambda}$ [26] for comparison. The Maxwell-type model describes well the time-dependent behavior of the FC for this simple liquid, which was expected from Fig. 2 showing good reproduction of the GK integral applying $\lambda(t)=\lambda_{M}(t)$ in Eq. (7). A slight distortion in the $\lambda_{M}$ profile comes from the nonvanishing time derivative at $t=0$. Note that $d \lambda /\left.d t\right|_{t=0}=0$ follows from Eq. (10) because the autocorrelation function is an even function in a stationary system. Figures 3(b) and 3(c) show $\lambda(t)$ for water confined either by graphene walls or by fcc walls at three different temperatures: 235,268 , and $360 \mathrm{~K}$. The profiles of the FC on both walls at $360 \mathrm{~K}$ (i.e., for liquid water above its melting point) look similar to those of the LJ liquid. However, for supercooled water, i.e., metastable liquid water below its melting point, $\lambda(t)$ cannot be described by the Maxwell-type model. It is known, for supercooled liquids, that the density relaxation is a two-step process with two characteristic decay times [46,47]: Here, one can see that the FC also decays with two characteristic times.

Conclusions. We have derived analytical expressions to connect the equilibrium fluctuations of the random force on the wall and the Navier friction coefficient (FC). The expressions are generic in the sense that they explicitly include the system size dependence and also the FC can be frequency dependent, which enabled us to address (i) the plateau issue on the evaluation of the FC and (ii) the non-Markovian behavior of the liquid-solid friction. For point (i) we found that the Green-Kubo integral of the random force autocorrelation has actually a plateau for the finite-sized systems and the plateau value has a clear hydrodynamic meaning. For point (ii) we evaluated the frequency- or time-dependent $\mathrm{FC}$ from equilibrium molecular dynamics simulation data for a Lennard-Jones (LJ) liquid and for water under different wall confinements and temperatures, without ambiguity due to the simulation system size. We showed that the Maxwell viscoelastic model is a fair approximation for the FC of LJ liquid on a fcc wall and that similarly a model with a single relaxation time can be applied for water on fcc and graphene walls at a high temperature, but a model with more than one time scale is required to describe the FC of supercooled water on both walls. Our theoretical framework opens the way to explore the frequency-dependent $\mathrm{FC}$ for a wide range of complex liquids by nondemanding atomistic simulations, whose system size may be small.

Acknowledgments. This work was financially supported by JSPS KAKENHI Grants No. JP21J20580, No. JP18K03929, and No. JP18K03978 and by the ANR, Project No. ANR16-CE06-0004-01 NECtAR. Y.Y. was also supported by JST CREST Grant No. JPMJCR18I1, Japan. L.J. was supported by the Institut Universitaire de France. 
[1] W. Sparreboom, A. van den Berg, and J. C. T. Eijkel, Principles and applications of nanofluidic transport, Nat. Nanotechnol. 4, 713 (2009)

[2] L. Bocquet and E. Charlaix, Nanofluidics, from bulk to interfaces, Chem. Soc. Rev. 39, 1073 (2010).

[3] H. Daiguji, Ion transport in nanofluidic channels, Chem. Soc. Rev. 39, 901 (2010).

[4] S. Faucher, N. Aluru, M. Z. Bazant, D. Blankschtein, A. H. Brozena, J. Cumings, J. P. de Souza, M. Elimelech, R. Epsztein, J. T. Fourkas, A. G. Rajan, H. J. Kulik, A. Levy, A. Majumdar, C. Martin, M. McEldrew, R. P. Misra, A. Noy, T. A. Pham, M. Reed et al., Critical knowledge gaps in mass transport through single-digit nanopores: A review and perspective, J. Phys. Chem. C 123, 21309 (2019).

[5] N. Kavokine, R. R. Netz, and L. Bocquet, Fluids at the nanoscale: From continuum to subcontinuum transport, Annu. Rev. Fluid Mech. 53, 377 (2021).

[6] L. Bocquet, Nanofluidics coming of age, Nat. Mater. 19, 254 (2020).

[7] L. Joly, C. Ybert, E. Trizac, and L. Bocquet, Hydrodynamics within the Electric Double Layer on Slipping Surfaces, Phys. Rev. Lett. 93, 257805 (2004).

[8] A. Ajdari and L. Bocquet, Giant Amplification of Interfacially Driven Transport by Hydrodynamic Slip: Diffusio-Osmosis and Beyond, Phys. Rev. Lett. 96, 186102 (2006).

[9] Y. Ren and D. Stein, Slip-enhanced electrokinetic energy conversion in nanofluidic channels, Nanotechnology 19, 195707 (2008).

[10] B. Radha, A. Esfandiar, F. C. Wang, A. P. Rooney, K. Gopinadhan, A. Keerthi, A. Mishchenko, A. Janardanan, P. Blake, L. Fumagalli, M. Lozada-Hidalgo, S. Garaj, S. J. Haigh, I. V. Grigorieva, H. A. Wu, and A. K. Geim, Molecular transport through capillaries made with atomic-scale precision, Nature (London) 538, 222 (2016).

[11] C. Navier, Mémoire sur les lois du mouvement des fluides, Mem. Acad. Sci. Inst. Fr. 6, 389 (1823).

[12] C. Neto, D. R. Evans, E. Bonaccurso, H.-J. Butt, and V. S. J. Craig, Boundary slip in Newtonian liquids: a review of experimental studies, Rep. Prog. Phys. 68, 2859 (2005).

[13] A. Maali and B. Bhushan, Measurement of slip length on superhydrophobic surfaces, Philos. Trans. R. Soc., A 370, 2304 (2012).

[14] W. Lei, M. K. Rigozzi, and D. R. McKenzie, The physics of confined flow and its application to water leaks, water permeation and water nanoflows: a review, Rep. Prog. Phys. 79, 025901 (2016).

[15] L. Bocquet and J. L. Barrat, Hydrodynamic Boundary Conditions and Correlation Functions of Confined Fluids, Phys. Rev. Lett. 70, 2726 (1993).

[16] L. Bocquet and J. L. Barrat, Hydrodynamic boundary conditions, correlation functions, and Kubo relations for confined fluids, Phys. Rev. E 49, 3079 (1994).

[17] J. Petravic and P. Harrowell, On the equilibrium calculation of the friction coefficient for liquid slip against a wall, J. Chem. Phys. 127, 174706 (2007).

[18] V. P. Sokhan and N. Quirke, Slip coefficient in nanoscale pore flow, Phys. Rev. E 78, 015301(R) (2008).

[19] J. S. Hansen, B. D. Todd, and P. J. Daivis, Prediction of fluid velocity slip at solid surfaces, Phys. Rev. E 84, 016313 (2011).
[20] K. Huang and I. Szlufarska, Green-Kubo relation for friction at liquid-solid interfaces, Phys. Rev. E 89, 032119 (2014).

[21] H. Oga, Y. Yamaguchi, T. Omori, S. Merabia, and L. Joly, Green-Kubo measurement of liquid-solid friction in finite-size systems, J. Chem. Phys. 151, 054502 (2019).

[22] P. Español, J. A. de la Torre, and D. Duque-Zumajo, Solution to the plateau problem in the Green-Kubo formula, Phys. Rev. E 99, 022126 (2019)

[23] J. A. De La Torre, D. Duque-Zumajo, D. Camargo, and P. Español, Microscopic Slip Boundary Conditions in Unsteady Fluid Flows, Phys. Rev. Lett. 123, 264501 (2019).

[24] H. Nakano and S.-i. Sasa, Microscopic determination of macroscopic boundary conditions in Newtonian liquids, Phys. Rev. E 99, 013106 (2019).

[25] H. Nakano and S.-i. Sasa, Equilibrium measurement method of slip length based on fluctuating hydrodynamics, Phys. Rev. E 101, 033109 (2020).

[26] T. Omori, N. Inoue, L. Joly, S. Merabia, and Y. Yamaguchi, Full characterization of the hydrodynamic boundary condition at the atomic scale using an oscillating channel: Identification of the viscoelastic interfacial friction and the hydrodynamic boundary position, Phys. Rev. Fluids 4, 114201 (2019).

[27] W. M. Slie, A. R. Donfor Jr., and T. A. Litovitz, Ultrasonic shear and longitudinal measurements in aqueous glycerol, J. Chem. Phys. 44, 3712 (1966).

[28] C. Masciovecchio, S. Santucci, A. Gessini, S. Di Fonzo, G. Ruocco, and F. Sette, Structural Relaxation in Liquid Water by Inelastic UV Scattering, Phys. Rev. Lett. 92, 255507 (2004).

[29] I. Omelyan, I. Mryglod, and M. Tokarchuk, Wavevector- and frequency-dependent shear viscosity of water: the modified collective mode approach and molecular dynamics calculations, Condens. Matter Phys. 8, 25 (2005).

[30] T. J. O'Sullivan, S. K. Kannam, D. Chakraborty, B. D. Todd, and J. E. Sader, Viscoelasticity of liquid water investigated using molecular dynamics simulations, Phys. Rev. Fluids 4, 123302 (2019).

[31] A. V. Straube, B. G. Kowalik, R. R. Netz, and F. Höfling, Rapid onset of molecular friction in liquids bridging between the atomistic and hydrodynamic pictures, Commun. Phys. 3, 126 (2020).

[32] J. C. F. Schulz, A. Schlaich, M. Heyden, R. R. Netz, and J. Kappler, Molecular interpretation of the non-Newtonian viscoelastic behavior of liquid water at high frequencies, Phys. Rev. Fluids 5, 103301 (2020).

[33] See Supplemental Material at http://link.aps.org/supplemental/ 10.1103/PhysRevResearch.3.L032019 for the unabridged derivation of the theory and additional computational information, which includes Refs. [26,34].

[34] L. Bocquet and J.-L. Barrat, On the Green-Kubo relationship for the liquid-solid friction coefficient, J. Chem. Phys. 139, 044704 (2013).

[35] As we show in the Supplemental Material [33], the frequency response characteristics of the FC and of the bulk viscosity are similar. This means that there is no bulk flow in the time scales where the high-frequency mode of the FC such as the elasticity dominates [26], and therefore one can neglect the frequency-dependent component of the bulk viscosity in the present analysis. 
[36] P. Mazur and I. Oppenheim, Molecular theory of Brownian motion, Physica (Amsterdam) 50, 241 (1970).

[37] S. Nishida, D. Surblys, Y. Yamaguchi, K. Kuroda, M. Kagawa, T. Nakajima, and H. Fujimura, Molecular dynamics analysis of multiphase interfaces based on in situ extraction of the pressure distribution of a liquid droplet on a solid surface, J. Chem. Phys. 140, 074707 (2014).

[38] K. Ogawa, H. Oga, H. Kusudo, Y. Yamaguchi, T. Omori, S. Merabia, and L. Joly, Large effect of lateral box size in molecular dynamics simulations of liquid-solid friction, Phys. Rev. E 100, 023101 (2019).

[39] J. L. F. Abascal, E. Sanz, R. García Fernández, and C. Vega, A potential model for the study of ices and amorphous water: TIP4P/Ice, J. Chem. Phys. 122, 234511 (2005).

[40] D. M. Huang, C. Cottin-Bizonne, C. Ybert, and L. Bocquet, Aqueous electrolytes near hydrophobic surfaces: Dynamic effects of ion specificity and hydrodynamic slip, Langmuir 24, 1442 (2008).

[41] K. Falk, F. Sedlmeier, L. Joly, R. R. Netz, and L. Bocquet, Molecular origin of fast water transport in carbon nanotube membranes: superlubricity versus curvature dependent friction, Nano Lett. 10, 4067 (2010).
[42] C. Herrero, G. Tocci, S. Merabia, and L. Joly, Fast increase of nanofluidic slip in supercooled water: the key role of dynamics, Nanoscale 12, 20396 (2020).

[43] The dimensions of the larger system were identical to those in Ref. [26]. Therefore the hydrodynamic height of the system $h$ of the larger system was taken from Ref. [26]. For the smaller system, $h$ was calculated considering that the hydrodynamic wall position should be identical to that of the larger system.

[44] L. Joly, G. Tocci, S. Merabia, and A. Michaelides, Strong coupling between nanofluidic transport and interfacial chemistry: How defect reactivity controls liquid-solid friction through hydrogen bonding, J. Phys. Chem. Lett. 7, 1381 (2016).

[45] C. Herrero, T. Omori, Y. Yamaguchi, and L. Joly, Shear force measurement of the hydrodynamic wall position in molecular dynamics, J. Chem. Phys. 151, 041103 (2019).

[46] W. Kob and H. C. Andersen, Testing mode-coupling theory for a supercooled binary Lennard-Jones mixture. II. Intermediate scattering function and dynamic susceptibility, Phys. Rev. E 52, 4134 (1995).

[47] P. Gallo, F. Sciortino, P. Tartaglia, and S. H. Chen, Slow Dynamics of Water Molecules in Supercooled States, Phys. Rev. Lett. 76, 2730 (1996). 